\title{
Évaluation de la mise en œuvre de la stratégie de gratuité des actes et des médicaments dans le district sanitaire de Bourem au Mali en 2014
}

\section{Evaluation of the implementation of the strategy of free acts and drugs in the health district of Bourem in Mali in 2014}

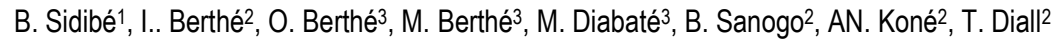

\begin{abstract}
1 Technicien Supérieur de Santé, Master II en sciences de la santé, Spécialité : Santé Publique,

Institut National de Formation en Science de la Santé Bamako, Mali ;

2 Master II Santé publique, Département d'Enseignement et de Recherche en Santé Publique et Spécialités, Faculté de Médecine et d'Odonto-Stomatologie, Université des Sciences, des Techniques et des Technologies de Bamako, Mali

${ }^{3}$ Directoion Générale de la Santé et de l'Hygiène Publique
\end{abstract}

\begin{abstract}
Résumé
Introduction : Instaurée dans les régions du nord par la lettre circulaire n0700 / MS-SG du Ministère de la santé, en date du 21 mai 2012, la politique de gratuité des actes et médicaments fait suite à la crise sécuritaire que le Mali a connu en 2012. Sa mise en œuvre dans le district sanitaire de Bourem a été confrontée à des difficultés d'où son évaluation. Matériel et méthodes : Une étude rétrospective a permis de collecter les données des rapports de 2011 et 2014 suivi d'un entretien avec les usagers / acteurs de la mise en œuvre soit 455 participants. Ces données ont permis de comparer certains indicateurs des périodes de 2011 et de 2014 avec un test de khi-deux d'une part et d'analyser celle de 2014 d'autre part. Résultats : Nous avions trouvé que les indicateurs de consultation médicale et prénatale premier contact de 2014 étaient meilleurs comparés à ceux de 2011. Le taux de rupture des médicaments du panier commun était élevé en 2014 , soit $45 \%$ contre un taux national de $20 \%$ pour la même année. Sur l'ensemble des usagers enquêtés, $65,5 \%$ trouvaient que la gratuité de la consultation curative était effective et $56,9 \%$ trouvaient que celle des médicaments l'était aussi. Conclusion : Au terme de l'étude, cette politique a été jugée peu satisfaisante par les agents de santé et les maires, par contre la majorité des usagers $(81,9 \%)$ et des ASACO $(76,9 \%)$ trouvait qu'elle doit continuer. Le suiviévaluation est indispensable pour la réussite de cette politique.
\end{abstract}

Mots clés: Évaluation, politique, gratuité, actes, médicaments, district sanitaire, Bourem

\footnotetext{
Abstract

Introduction: Introduced in the northern regions by the Ministry of Health's Circular Letter No. 0700 / MS-SG, in $21^{\text {th }}$ May, 2012, the policy of free medical services and drug delivery following the security crisis that Mali experienced in 2012. Its implementation in the health district of Bourem was confronted with difficulties hence its evaluation. Material and methods: A retrospective
}

study was implemented to collect data from 2011 and 2014 reports followed by an interview with the users / stakeholders of the implementation including a total of 455 participants. The data made compared the period of 2011 and 2014 using chi-square test. Results: We found that 2014 medical and prenatal consultation indicators were better compared to 2011. The drug shortage rate (45\%) for common basket medications was high in 2014 compared to the national rate of $20 \%$ for the same annual period. Of all the users surveyed, $65.5 \%$ found that free consultation was effective and $56.9 \%$ found that the medication was also free. Conclusion: At the end of the study, this policy was deemed unsatisfactory by health workers and mayors, while the majority of users $(81.9 \%)$ and ASACOs (76.9\%) felt that it should continue. Therefore, monitoring and evaluation is essential for the success of this policy.

Keywords: Evaluation, policy, free, acts, drugs, health district, Bourem

\section{Introduction}

A travers le monde, la gratuité dans le secteur de la santé est appliquée sous différentes formes selon les contextes. Aux États-Unis d'Amérique le « kid care » chez les enfants et "MedAid» chez les personnes âgées [1] ; au Liberia, les soins de santé primaire gratuits pour tous depuis 2007 [2].

Le Mali a gratifié la prise en charge de certaines pathologies et actes médicaux dans les établissements de santé, notamment :

- Soins et examens paracliniques pour les maladies chroniques [3],

- Prise en charge de la césarienne [4],

- Prise en charge du VIH-Sida [5],

- Prise en charge du paludisme chez les enfants de moins de cinq ans et chez la femme enceinte [6].

Suite à la crise sécuritaire qu'a connu le Mali en 2012, les districts sanitaires touchés y compris Bourem ont bénéficié de la gratuité des actes et médicaments suivant la lettre circulaire $n^{\circ}$ 0700/MS-SG en date du 21 mai 2012 du Ministère de la santé et adressé aux partenaires techniques et financiers du Ministère de la santé, aux organisations non gouvernementales (ONG) intervenant dans le secteur de la santé des zones touchées [7].

Dans sa mise en œuvre dans le district sanitaire de Bourem, les acteurs locaux et usagers ont signalé des difficultés telles que :

- la rupture fréquente des médicaments essentiels et consommables dans les établissements de santé, 
- l'absence de couverture totale de tous les centres de santé communautaires par cette politique,

- le recouvrement parallèle des actes par les agents de santé,

- la vente des médicaments par les agents de santé dans les établissements de santé,

- le retard dans la transmission des rapports d'activités des Centre de santé communautaires (CSCom) au Centre de santé de référence (CSRéf).

Au regard de ces constats, cette étude a été initié pour évaluer la mise en œuvre de la gratuité des actes et des médicaments dans le district sanitaire de Bourem en 2014.

\section{Matériel et méthodes}

Cadre de l'étude

L'étude a été réalisée dans le district sanitaire de Bourem, situé à $95 \mathrm{~km}$ de la région de Gao au Mali (Figure1).

\section{Schéma d'étude}

II s'agissait d'une étude transversale à visée évaluative basée sur l'analyse des rapports. L'aspect prospectif a concerné l'entretien avec les acteurs de la mise en œuvre.

Les données ont été collectées du 24 novembre 2016 au 11 janvier 2017.

\section{Échantillonnage}

Les participants de la phase d'entretien étaient les usagers et les acteurs de la mise en œuvre de la politique de gratuité en 2014 ayant accepté les conditions de l'entrevu.

La taille de l'échantillon des usagers a été déterminée par la formule de Daniel SHWARTZ avec $p=0,47$ correspondant au taux moyen de consultation curative nouveau cas en 2014 dans le district sanitaire et i le niveau de précisons à 0,05 .

La technique d'échantillonnage par grappe à quatre degrés a permis de sélectionner 422 usagers dans 7 aires de santé, 21 villages et 420 ménages.

Pour les autres cibles, le choix a été raisonné et au total 14 agents de santé, 13 membres d'ASACO, 4 maires et 2 coordinateurs d'ONG ont été enrôlés.

\section{Analyse statistique}

Le logiciel Epi-info 3.5.4 et Microsoft office Excel 2002 ont été utilisés pour analyser les données. Le test de khideux a été utilisé pour comparer les indicateurs des années 2011 et 2014.

Considérations éthiques

L'étude a respecté la réglementation en vigueur en matière d'éthique. Les objectifs et but de l'étude ont été expliqués aux participants qui ont donnés chacun leur consentement éclairé, volontaire et écrit. Les autorités ont été informées et leurs autorisations ont contribué à la réalisation.

\section{Résultats}

\section{Analyse comparative des indicateurs}

La comparaison des taux des différents indicateurs des années 2011 et 2014 avec le test de khi-deux a permis d'avoir une différence significative en faveur de 2014 pour les indicateurs suivants: Consultation Prénatale (CPN) : CPN1, CPN3; Consultation curative ; Prévalence contraceptive; Moustiquaires chez les femmes enceintes; Moustiquaires chez les enfants de moins 5 ans. Pour toutes ces variables la valeur de khideux était supérieure à 3,84 (tableau I).

Le taux de rupture de stock de médicaments du panier commun était de $11 \%$ en 2011 contre 45\% en 2014.

\section{Profil des interviewés}

Pour l'ensemble des personnes enquêtées, le sexe féminin était le plus dominant avec $52,5 \%$ (tableau II) et la tranche d'âge la plus représentée était celle de 20 à 39 ans (tableau III), les paysans représentaient 77,3\% (tableau IV).

\section{Appréciation de l'effectivité de la gratuité}

Sur l'ensemble des usagers interviewés, $65,5 \%$ ont affirmé l'effectivité de la gratuité des consultations médicales et $34,5 \%$ le contraire, $56,9 \%$ ont affirmé l'effectivité de la gratuité des médicaments et $82,9 \%$ ont affirmé l'effectivité de la gratuité des soins. Sur l'ensemble des usagers ayant considéré la gratuité des actes non effective, $84,7 \%$ ont affirmé avoir reçu un ticket.

Partenariat Association de santé communautaire (ASACO)-Collectivités-État-PTF

Aucun des 4 Maires (élus communaux) enquêtés n'avait reçu de document officiel relatif à la politique de gratuité dans les établissements. L'ensemble des 4 élus communaux et des 13 présidents d'ASACO enquêtés ont affirmé l'existence d'un protocole d'accord avec les partenaires intervenant dans la mise en œuvre de la politique de gratuité. Sur l'ensemble des ASACO enquêtées, 53,8\% n'étaient pas satisfaits des livraisons des médicaments par rapports aux commandes contre $46,2 \%$ de satisfaits.

Opinion des usagers et des acteurs sur la continuité de la gratuité

La majorité des usagers et des membres d'ASACO enquêtés (soit respectivement $81,9 \%$ et $76,9 \%$ ) ont opté pour la continuité de la gratuité totale. Seulement $42,9 \%$ du personnel de santé et $25 \%$ des élus communaux enquêtés ont opté pour sa continuité (tableau VIII).

\section{Discussion}

Certains aspects des soins et services n'ont pas été pris en compte par cette étude, notamment la dotation des établissements de santé en équipement, la réhabilitation des infrastructures. La non disponibilité d'études similaires dans la revue de la littérature n'a pas permis une discussion plus large. 
Nos résultats ont trouvé que la politique de gratuité des soins et des médicaments soutenu par l'état et ses partenaires a positivement influencé la fréquentation des établissements de santé. Les indicateurs de CPN1, CPN3, de la consultation curative, de la prévalence contraceptive, de l'accès aux moustiquaires chez les femmes enceintes et chez les enfants de moins de 5 ans étaient significativement supérieur en 2014 qu'en 2011. L'équipe de Médecins du monde au Niger en juillet 2011 a trouvé que la gratuité des prestations à l'endroit des femmes en âge de procréer, des femmes enceintes et des enfants de moins de 5 ans a contribué activement à l'amélioration des indicateurs [2]. Nous avons trouvé un taux de rupture de stock de médicaments de panier commun plus élevé en période de gratuité qu'en période sans gratuité soit respectivement $45 \%$ en 2014 et $11 \%$ en 2011. Ce résultat est similaire à celui de Sanogo D. et al sur l'évaluation du processus de mise en œuvre du système de gratuité du traitement du paludisme chez les enfants de moins de cinq ans dans le district de Bamako en 2010, qui a révélé les ruptures fréquentes des médicaments dans les centres de santé [8].

Parmi les usagers interviewés $34,5 \%$ affirmait avoir payé de l'argent pour la consultation, 43,1\% affirmait avoir payé les médicaments et $17,1 \%$ affirmait avoir payé les soins (perfusion, injection, pansement). Ce non-respect de la politique pourrait être expliqué par :

- l'insuffisance d'information et de sensibilisation des acteurs,

- une rupture des médicaments essentiels dans les dépôts des établissements de santé.

L'étude de Sanogo D. et al. a trouvé en 2010 à Bamako le même résultat concernant l'information des parents des bénéficiaires [8].

Notre évaluation avait trouvé qu'aucune collectivité communale enquêtée n'avait reçu un document officiel sur l'instauration de la politique de gratuité des actes et des médicaments. Cela peut être l'une des raisons de la non application de la politique dans tous les établissements sanitaires.

La majorité des ASACO $(92,3 \%)$ et des élus communaux $(75 \%)$ affirmait le respect des engagements des protocoles d'accord par les ONG. L'ensemble des ONG affirmait le respect total des engagements des protocoles d'accord par les ASACO et mairies.

La majorité des usagers interviewés $(92,4 \%)$ était satisfait de l'accueil par les agents dans les établissements de santé. Ce résultat corrobore avec l'étude de Jean HMB et al. sur la satisfaction des usagers du service de pédiatrie à l'hôpital de Grâce Children à Delmas (Haïti) en août 2007 qui a trouvé que $88 \%$ des usagers déclare être très bien accueilli par le staff de l'hôpital [9].

Les principales causes évoquées par ceux non satisfaits de l'accueil étaient le temps d'attente prolongé $(51,7 \%)$, la négligence $(31,1 \%)$, l'arrogance $(10,3 \%)$ et la non disponibilité d'agent de santé $(6,9 \%)$.
En ce qui concerne la disponibilité des médicaments, parmi les usagers n'ayant pas trouvé de médicaments dans les dépôts de vente, $80 \%$ d'entre eux ont affirmé avoir acheté le reste des médicaments dans la pharmacie externe (office, dépôt privé) et $20 \%$ au près des agents de santé non habilité à la vente des médicaments.

\section{Conclusion}

Cette étude a montré que la politique de gratuité des actes et des médicaments améliore significativement la fréquentation des établissements de santé avec un effet négatif sur la qualité des soins. Raison pour laquelle elle a été jugé peu satisfaisante. Ainsi le suivi-évaluation continu serait une étape importante pour la bonne marche de cette politique.

\section{Contributions des auteurs}

$\mathrm{BS}^{3 / 4}$ a initié le draft, corrigé le fond et a traduit le résumé. IB a participé à toutes les phases de la rédaction du document. $\mathrm{BS}^{2}$ a collecté les données et a relu le travail. ANK a relu et corrigé le document. TD a supervisé la rédaction de ce document, il l'a relu. Tous les auteurs ont relu et approuvé la version finale du manuscrit.

\section{Conflit d'intérêt}

Les auteurs ne déclarent aucun conflit d'intérêt.

\section{Références}

1. Becker G. al, child endowment and the quantity and quality of children, Journal of Political Economy $n^{\circ} 84$, publié en 1976, p.3-7.

2. Médecins du Monde, gratuité des soins au Niger, une option payante à consolider, Edition juillet 2011, p.8-13

3. Présidence Secrétariat général du gouvernement du Mali, décret $n^{\circ} 02-311 / P-R M$ du 04 juin 2002 fixant le régime de la rémunération des actes médicaux et de l'hospitalisation dans les établissements publics de santé.

4. Primature, Secrétariat général du gouvernement du Mali, décret $n^{\circ}$ 05-350/P-RM du 04 aoút 2005 relatif à la prise en charge gratuite de la césarienne, articles $2,3,4,5$.

5. Présidence de la République du Mali, Haut conseil de lutte contre le VIH /SIDA, déclaration de politique de lutte contre le VIH/SIDA, adopte par le Gouvernement le 07 avril 2004, p.17.

6. Primature, Secrétariat général du gouvernement de la République du Mali, décret $n^{\circ} 10-628 / P-R M ~ d u ~ 29$ novembre 2010 portant gratuité des moyens de prévention et de traitement du paludisme chez l'enfant de moins de cinq ans et chez la femme enceinte dans les établissements de santé, articles 1 , 2. 
7. Ministère de la santé du Mali, secrétariat général, lettre n0700 / MS-SG du 21 mai 2012, relative à la gratuité des actes et médicaments, p.1.

8. SANOGO D., Évaluation du processus de mise en œuvre du système de gratuité du traitement du paludisme chez les enfants de moins de cinq ans dans le district de Bamako, École des hautes études en santé publique, 2010, p31-46.

9. Jean HMB. et al, Étude de la satisfaction des usagers du service de pédiatrie de l'Hôpital Grâce Children, Faculté de médecine/université d'état d'Haïti, Aoút 2007, p.26.

Liste des tableaux :

Tableau I: Comparaison des indicateurs de 2011 et 2014 du district sanitaire de Bourem

\begin{tabular}{|c|c|c|c|c|}
\hline Indicateurs & $\begin{array}{l}\text { Taux (\%) } \\
\text { en } 2011\end{array}$ & $\begin{array}{l}\text { Taux (\%) } \\
\text { en } 2014\end{array}$ & Khi2 & $p$ \\
\hline CPN1 & 69 & 83 & 5,37 & 0,02 \\
\hline CPN3 & 23 & 44 & 9,90 & 0,001 \\
\hline Accouchement & 20 & 22 & 0,12 & 0,728 \\
\hline $\begin{array}{ll}\text { assisté } & \text { au } \\
\text { centre } & \text { de } \\
\text { santé } & \end{array}$ & & & & \\
\hline $\begin{array}{l}\text { Accouchement } \\
\text { par personnel } \\
\text { qualifié }\end{array}$ & 65 & 67 & 0,09 & 0,76 \\
\hline $\begin{array}{l}\text { Consultation } \\
\text { curative }\end{array}$ & 32 & 47 & 4,71 & 0,03 \\
\hline Césarienne & 0,76 & 0,97 & 2,16 & 0,14 \\
\hline $\begin{array}{l}\text { Prévalence } \\
\text { contraceptive }\end{array}$ & 1,52 & 0,92 & 45,47 & 0,000 \\
\hline MII FE & 69 & 51 & 6,75 & 0,000 \\
\hline $\begin{array}{l}\text { MII enfant de } \\
\text { moins d' } 1 \text { an }\end{array}$ & 85 & 50 & 27,92 & 0,000 \\
\hline VAT 2 FE & 69 & 74 & 0,61 & 0,43 \\
\hline
\end{tabular}

Tableau II: Répartition en fonction du sexe, des personnes enquêtées sur la gratuité dans le district sanitaire de Bourem en 2014 ( $n=414)$.

\begin{tabular}{|c|c|c|c|c|}
\hline \multirow{2}{*}{$\begin{array}{l}\text { Personnes } \\
\text { enquêtées }\end{array}$} & \multicolumn{3}{|c|}{ Sexe } & \multirow[t]{2}{*}{ Tota } \\
\hline & & Féminin & Masculin & \\
\hline \multirow[t]{2}{*}{ Usagers } & $\mathrm{n}$ & 201 & 182 & 383 \\
\hline & $\%$ & 52,5 & 47,5 & 100 \\
\hline \multirow{2}{*}{$\begin{array}{l}\text { Membres } \\
\text { d'ASACO }\end{array}$} & $\mathrm{n}$ & 0 & 13 & 13 \\
\hline & $\%$ & 0 & 100 & 100 \\
\hline \multirow[t]{2}{*}{ Élus } & $n$ & 0 & 4 & 4 \\
\hline & $\%$ & 0 & 100 & 100 \\
\hline \multirow{2}{*}{$\begin{array}{l}\text { Personnel } \\
\text { de santé }\end{array}$} & $\mathrm{n}$ & 1 & 13 & 14 \\
\hline & $\%$ & 7,1 & 92,9 & 100 \\
\hline
\end{tabular}

Tableau III : Répartition en fonction de la profession des usagers enquêtés sur la gratuité dans le district sanitaire de Bourem en 2014 ( $n=383$ )

\begin{tabular}{lll}
\hline Profession & $\mathbf{n}$ & $\%$ \\
\hline Artisans & 28 & 1,6 \\
\hline Paysans & 296 & 13,3 \\
\hline Fonctionnaire & 15 & 27,7 \\
\hline Commerçant & 9 & 27,7 \\
\hline Elève & 30 & 15,1 \\
\hline Sans profession & 5 & 9,4 \\
\hline Total & 383 & 100 \\
\hline
\end{tabular}

Tableau IV: Répartition selon l'âge des usagers enquêtés sur la gratuité dans le district sanitaire de Bourem en 2014 ( $n=383)$

\begin{tabular}{|lll|}
\hline Tranche d'âge & $\mathbf{n}$ & $\%$ \\
\hline$<\mathbf{1 0}$ ans & 6 & 1,6 \\
\hline $\mathbf{1 0}-\mathbf{1 9}$ ans & 51 & 13,3 \\
\hline $\mathbf{2 0}-\mathbf{2 9}$ ans & 106 & 27,7 \\
\hline $\mathbf{3 0}-\mathbf{3 9}$ ans & 106 & 27,7 \\
\hline $\mathbf{4 0}-\mathbf{4 9}$ ans & 58 & 15,1 \\
\hline $\mathbf{5 0}-\mathbf{5 9}$ ans & 36 & 9,4 \\
\hline $\mathbf{6 0}$ et plus & 20 & 5,2 \\
\hline Total & $\mathbf{3 8 3}$ & $\mathbf{1 0 0}$ \\
\hline
\end{tabular}

Tableau V : Répartition des personnes enquêtées en fonction de leur opinion sur la continuité de la gratuité totale dans le district sanitaire en 2014 ( $n=414)$

\begin{tabular}{lllllll}
\hline Personnes enquêtées & \multicolumn{3}{c}{$\mathbf{n}$} & \multicolumn{3}{c}{$\%$} \\
\cline { 2 - 7 } & Oui & Non & Total & Oui & Non & Total \\
Usagers & 314 & 69 & $\mathbf{3 8 3}$ & 81,9 & 18,1 & 100 \\
Membres d'ASACO & 10 & 3 & 13 & 76,9 & 23,1 & 100 \\
Personnel de santé & 6 & 8 & 14 & 42,9 & 57,1 & 100 \\
Élus communaux & 1 & 3 & 14 & 25 & 75 & 100 \\
\hline
\end{tabular}




\section{MALI SANTE PUBLIQUE 2019 SOMASAP}

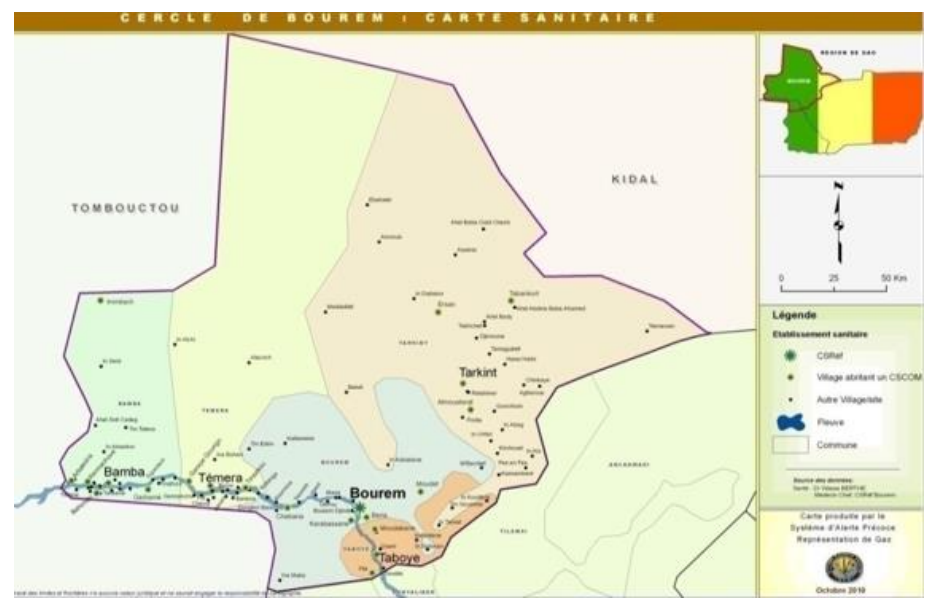

Figure 1 : Carte sanitaire de Bourem en 2014 